\title{
Mesospheric observations with the EISCAT UHF radar during polar cap absorption events: 3. Comparison with simultaneous EISCAT VHF measurements
}

\author{
P. N. Collis ${ }^{1}$, M. T. Rietveld ${ }^{2}$ \\ ${ }^{1}$ EISCAT Scientific Association, Box 812, S-981 28 Kiruna, Sweden \\ ${ }^{2}$ EISCAT Scientific Association, Ramfjordmoen, N-9020 Tromsdalen, Norway
}

Received: 29 September 1997 / Revised: 14 April 1998 / Accepted: 17 April 1998

\begin{abstract}
Mesospheric observations were obtained by the EISCAT UHF and VHF radars during the solar proton event of March 1990. We present the first comparison of incoherent-scatter spectral measurements from the middle mesosphere using simultaneous, colocated observations by the two radars. VHF spectra observed with a vertical antenna were found to be significantly narrower than model predictions, in agreement with earlier UHF results. For antenna pointing directions that were significantly away from the vertical, the wider VHF radar beam gave rise to broadening of the observed spectra due to vertical shears in the horizontal wind. In this configuration, UHF spectral measurements were found to be more suitable for aeronomical applications. Both radar systems provide consistent and reliable estimates of the neutral wind. Spectral results using both the multipulse and pulse-topulse schemes were intercompared and their suitability for application to combined mesosphere - lower thermosphere studies investigated.
\end{abstract}

Key words. Mesophere $\cdot$ Lower thermosphere · EISCAT UHF radar · EISCAT VHF radar

\section{Introduction}

Incoherent-scatter radars (ISRs) have been used for probing the Earth's ionosphere since the early 1960s (Evans, 1969; Bauer, 1975, Beynon and Williams, 1978). Altitudes near the lower boundary of the ionosphere present a difficult target for an ISR due to the low degree of ionisation normally encountered there. The region considered here is below about $95 \mathrm{~km}$ altitude and

Correspondence to: P. N. Collis constitutes the ionospheric D-region; in terms of the neutral atmosphere these heights are in the middle and upper mesosphere and lower thermosphere. The ability to apply the incoherent-scatter technique requires the probing radar wavelength to be greater than the Debye length of the scattering medium. For the low plasma densities in the D-region the longer wavelength of the EISCAT VHF radar $(1.34 \mathrm{~m})$ is expected to be more appropriate than that of the EISCAT UHF radar $(0.32 \mathrm{~m})$. The capability of simultaneous, dual-radar operation allows the performances of the two systems to be compared.

The earliest ISR spectral measurements from mesospheric heights at auroral latitudes were with the EISCAT UHF radar in 1982 (Kofman et al., 1984). Since then, the techniques have been improved and such measurements are now routine (Collis and Röttger, 1990). The initial EISCAT observations of mesospheric spectra using essentially the same experiment scheme as the measurements to be discussed here were obtained in the summer of 1985 with the $933-\mathrm{MHz}$ UHF radar (E. Turunen et al., 1988; Collis et al., 1988). Details of the experiment algorithm, termed GEN-11, have been given by T. Turunen (1986) and are described later. The VHF radar $(224 \mathrm{MHz})$ became available for similar measurements in 1987 (Hall et al., 1987).

Although the two EISCAT radar systems have separately shown themselves to be capable of providing spectral measurements from the lowest part of the ionosphere, the simultaneous operation of both radars is also routinely reliable. In this paper we describe the first such dual-radar observations using the same pulse modulation experiment over the same middle-mesosphere height range. These measurements took advantage of enhanced low-altitude ionisation, due to an ongoing solar proton event (SPE), to probe as low as $50 \mathrm{~km}$ altitude. This is much lower than for any other previously reported EISCAT VHF spectral measurements. A comparison is also made between VHF spectral measurements with the GEN-11 scheme and with the UHF multipulse scheme, which at times 
allowed observations overlapping in altitude in the upper mesosphere. This study is a continuation of the earlier works reported by Collis and Rietveld (1990) and Rietveld and Collis (1993).

\section{Measurement techniques and data analysis}

Standard analysis of mesospheric incoherent-scatter spectra is usually based on fitting a curve of Lorentzian shape to the measured spectra. This curve is fully defined by the four quantities of spectral width, spectral amplitude, displacement from zero frequency (i.e. Doppler shift) and a base level representing noise other than the wanted ion line (Mathews, 1986). An alternative is to fit the Fourier transform, which is an exponential curve, to the autocorrelation functions (ACFs). Details of how this method is applied to EISCAT data have been described by Rietveld and Collis (1993). Electron density and line-of-sight ion velocity are the only geophysical parameters that may be routinely determined from an initial analysis of the spectra. The spectral width is a scalar quantity depending on four geophysical parameters: ion-neutral collision frequency (or, alternatively, neutral air density), mean positive ion mass, neutral temperature and number density ratio of negative ions to electrons (see e.g. Collis and Röttger, 1990). Determination of any of these quantities needs information about the others, either from models or from independent measurements. A particularly promising method of interpreting the observations is with the use of detailed ion-chemical models (E. Turunen, 1993, 1996).

Spectra from the lower part of the altitude region probed by EISCAT pulse-to-pulse correlation measurements are easily encompassed within the bandwidths of the experiments reported here $( \pm 225 \mathrm{~Hz}$ with resolution $11 \mathrm{~Hz}$ for UHF, and $\pm 154 \mathrm{~Hz}$ with resolution $7 \mathrm{~Hz}$ for VHF). The available bandwidth is set by the interpulse period (IPP) of the transmitted pulses, which also defines the lag spacing of the measured ACFs for pulse-to-pulse correlation. For the UHF experiment the IPP was $2.222 \mathrm{~ms}$, and for the VHF, $3.24 \mathrm{~ms}$. Sampling these pulses at any given height thus leads to total bandwidths of \pm 225 and $\pm 154 \mathrm{~Hz}$, respectively. The frequency resolution is just the inverse of the longest lag measured by the experiment. As GEN-11 provides ACFs with 43 lags, the frequency resolution is $(43 \times \text { IPP })^{-1} \mathrm{~Hz}$.

Figure 1 illustrates the spectral widths expected for mesospheric measurements at the two operating frequencies, assuming two different mean atmosphere models [following Eq.(2) of Rietveld and Collis (1993)]. Note that this diagram supersedes Fig. 2 of Collis and Röttger (1990), which was incorrectly drafted, and extends to lower altitudes than the published correct version (Collis and Röttger, 1991).

At some height in the upper mesosphere, the spectral width of the backscattered signal becomes wider than the employed bandwidth and the pulse-to-pulse technique cannot be applied at altitudes above this. Since the

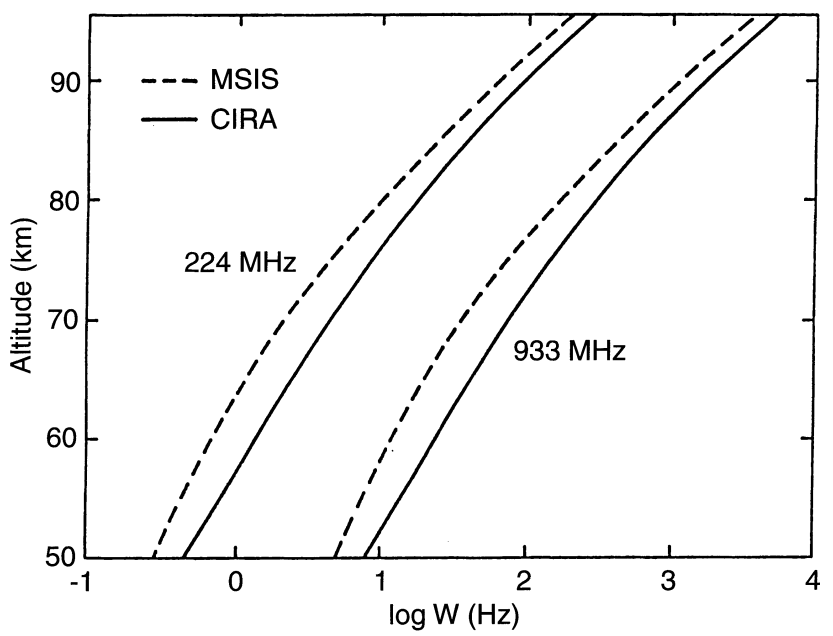

Fig. 1. Calculated incoherent-scatter spectral widths $(W)$ in the upper mesosphere for the EISCAT UHF (933 MHz) and VHF (224 MHz) radars, for the CIRA-72 (full lines) and extrapolated MSIS-83 (broken lines) atmospheric models, assuming no negative ions present

spectral width at a given height will vary with time [especially with season, see Fig. 4 of Rietveld and Collis (1993)] according to variations in the neutral and ionised atmosphere, the upper height limit for application of the technique will vary also. For the above bandwidths, this upper limit is typically $80-85 \mathrm{~km}$ for UHF measurements and about $95-98 \mathrm{~km}$ for VHF. This height regime is also close to the lower altitude limit where the standard multipulse technique may be successfully applied (T. Turunen and Silén, 1984; Kofman and Lathuillere, 1985; T. Turunen, 1986). Hence in experiments where pulse-to-pulse and multipulse measurements are obtained, either simultaneously or close in time to each other, it should be possible to compare directly the observed spectra when the height ranges of the two sets of data overlap. Such a comparison could serve to provide spectral measurements throughout both the mesosphere and lower ionosphere for particular studies (normal practice has been to acquire one or the other). It could also allow a detailed comparison of the observations from the two techniques in the height region of overlap, permitting a more critical evaluation of the results than could be afforded by using one technique alone.

The GEN-11 pulse-to-pulse correlation algorithm (T. Turunen, 1986) transmits Barker-coded double pulses of $7-\mu \mathrm{s}$ baud and provides spectra in 42 gates at $1.05-\mathrm{km}$ intervals in range starting at $70 \mathrm{~km}$ range. Clutter contaminates the first five gates; the first uncluttered signals are returned from $75 \mathrm{~km}$ range. During the experiments to be reported, the energetic proton precipitation associated with the SPEs caused ionisation down to altitudes of about $50 \mathrm{~km}$. Signals were obtained from these altitudes by tilting the antennas to lower elevations, away from the nominal vertical pointing direction.

The multipulse measurements started at $90 \mathrm{~km}$ range in the experiments described below, which translates to $78 \mathrm{~km}$ altitude for the lowest antenna elevation $\left(60^{\circ}\right)$. Under normal circumstances, the first one or two 


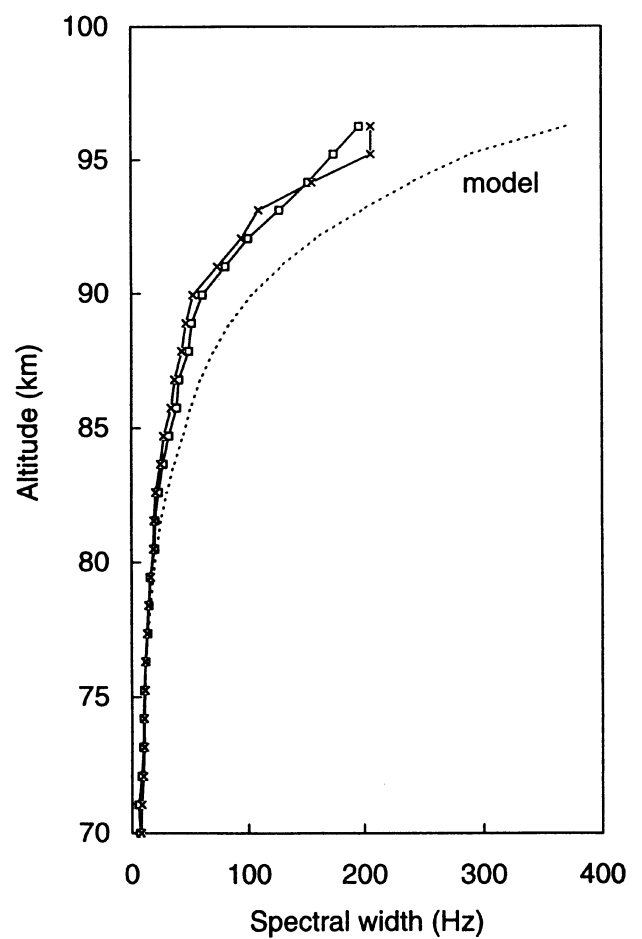

Fig. 2. The left panel shows measurements of spectral width by the EISCAT VHF radar with a vertically pointing antenna compared with model values. The profile with open square symbols $(\square)$ is from the results of fitting exponential curves to the ACFs while that with crosses (x) shows the results of fitting Lorentzian curves to the spectra.

multipulse gates (resolution $2.7 \mathrm{~km}$ in range) usually contain too much ground clutter to be useful, but under strong signal conditions (such as during SPEs) this effect is not usually a severe limitation. In the following we present a comparison of multipulse and pulse-to-pulse results, as well as of the simultaneous observations of pulse-topulse spectra on both UHF and VHF frequencies.

\section{Geophysical conditions and operational details}

The measurements we report were obtained during periods of solar proton events causing conditions of polar cap absorption (PCA) and associated large ionisation rates at low altitudes in the polar and auroral mesosphere. Such events were relatively numerous during the maximum phase of solar cycle 22 . The EISCAT UHF radar made successful measurements during seven of the eight most intense SPEs during 1989 (Collis and Rietveld, 1990). We will use data from two of these 1989 events (23 October and 1 December) when pulse-to-pulse and multipulse measurements were obtained. In addition, results will be presented from a subsequent SPE, on 20 March 1990, when simultaneous UHF and VHF pulse-to-pulse spectra were recorded, together with simultaneous UHF multipulse and VHF pulse-to-pulse data from a slightly later time on the same day.

Details of the EISCAT operations during the SPEs in 1989, as well as details of the events themselves, have been presented by Collis and Rietveld (1990). The March 1990 SPE commenced at 0905 UT on 19 March, reached a peak flux $(>10 \mathrm{MeV})$ of $950 \mathrm{~cm}^{-2} \mathrm{~s}^{-1} \mathrm{sr}^{-1}$ at 2315 UT on 19 March and decreased below $10 \mathrm{~cm}^{-2} \mathrm{~s}^{-1} \mathrm{sr}^{-1}$ at $1050 \mathrm{UT}$ on 21 March (Solar Geophysical Data, 1990). The peak 30-MHz radio absorption recorded in the polar cap (at Thule, $L=250$ ) during this event was $9.1 \mathrm{~dB}$ at 1615 UT on 19 March. EISCAT's GEN-11 VHF operations commenced at 0900 UT on 20 March and finished at 1600 UT on 22 March. The antenna was initially pointed vertically, but was subsequently lowered in elevation to cover lower altitudes. Substantial increases in electron density were observed at heights above about $50 \mathrm{~km}$ (the lower limit of the measurements) during the daytime hours on 20 March (from the start at 09 UT until about 19 UT), and above about $60-65 \mathrm{~km}$ on 21 March (from about 03 to 19 UT). The densities on 21 March were of smaller magnitude than the previous day due to the decreased intensity of the proton flux. On 22 March there was no obvious excess ionisation in the mesosphere.

With the VHF experiment successfully in operation on 20 March, the same experiment scheme was started and operated simultaneously on the UHF system. Both antennas were lowered in elevation so that the first uncluttered signal gates at $75 \mathrm{~km}$ range just encompassed the lower border of the excess ionisation in the mesosphere near $50 \mathrm{~km}$ altitude. This required elevations of $45^{\circ}$; the antennas were pointed parallel and due north, thereby measuring approximately the same 
ionospheric volumes (the beams have slightly different cross-sections). Due to technical problems, raw UHF data from the GEN-11 experiment could only be recorded for the interval 1240-1440 UT on 20 March. At 1530 UT, the UHF experiment mode was changed to CP-2-D, which included a short-pulse power profile with a lowest height of $59 \mathrm{~km}$, together with a five-pulse multipulse with a lowest height of $78 \mathrm{~km}$. This operation continued until 16 UT on 22 March.

\section{Comparison of results from different measurement schemes}

\subsection{Vertically directed VHF radar observations}

The first half-hour of VHF radar operations on 20 March 1990 used a vertical antenna. Results from this period allowed the truest estimate of spectral width because, as to be described, the spectra became broadened when the antenna was later pointed away from the vertical. Figure 2 shows two estimates of the mean profile of spectral width from this interval, from independent fits to both the spectra and the ACFs as discussed by Rietveld and Collis (1993). These profiles were constructed from fifteen independent 2-min averages to exclude as far as possible any broadening due to changes in the vertical motion of the atmosphere on time-scales of $2 \mathrm{~min}$ or longer. The profile of the ratio of the two widths indicates a tendency for the ACF exponential fit results to be increasingly larger than the spectral Lorentzian fits with increasing altitude, i.e. with increasing spectral width. The ratio is rather constant with a value of $\sim 1.1$ between heights of $\sim 80$ and $93 \mathrm{~km}$.
This result is similar to that from a like comparison made with data from the EISCAT UHF radar by Rietveld and Collis (1993). There it was explained that the difference arises from aliasing effects when fitting in the frequency domain (though total power and velocity estimates are not affected). The averaging in Fig. 2 leads to smaller differences between the two fitting methods than the UHF analysis, as the latter used results from individual 2-min integrations without subsequent averaging. The large variations in the ratio near the upper and lower extremes of the displayed altitude range in Fig. 2 (right panel) are probably related to the limited spectral bandwidth of the experiment, and limited frequency resolution, respectively.

Also shown in Fig. 2 is a model spectral width profile calculated from the CIRA (1972) atmosphere. Note that the observed values are significantly smaller than the model ones for altitudes above $\sim 76 \mathrm{~km}$. This discrepancy is consistent with similar results from the EISCAT UHF radar (Hansen et al., 1991; Rietveld and Collis, 1993), though the cause of the difference is still a matter of speculation. For altitudes below $\sim 76 \mathrm{~km}$ the measured spectral widths are close to the minimum resolvable frequency with this experiment $(7 \mathrm{~Hz})$, so we cannot comment definitively on the true relation between the observed and model widths.

\subsection{Obliquely directed VHF radar observations}

After the first half-hour of measurements on 20 March 1990 , the VHF antenna was lowered in elevation, first to $50^{\circ}$ for $20 \mathrm{~min}$, then to $45^{\circ}$ where it remained for the rest of the daytime measurements. The right panel of Fig. 3

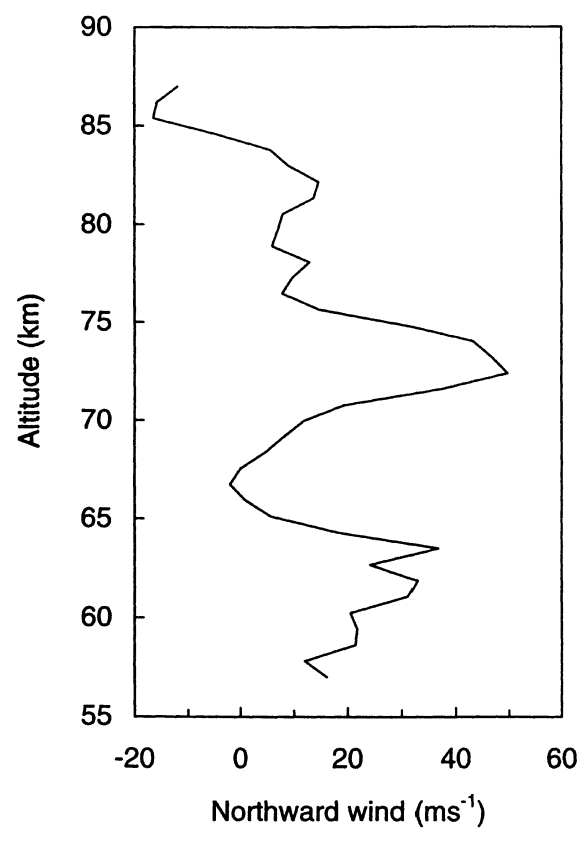

Fig. 3. Left: altitude profile of horizontal meridional wind measured with an antenna elevation angle of $50^{\circ}$ at 0952 UT, 20 March 1990. The results are from analyses of 2-min integrations of the raw VHF

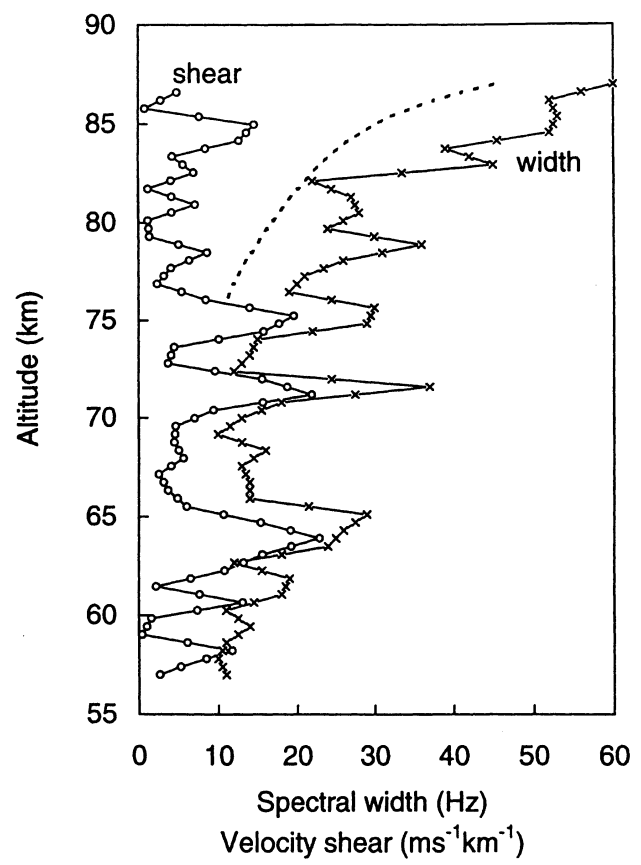

data. Right: altitude profile of VHF spectral width (x) and velocity shear (o). The smooth broken curve shows the model values from Fig. 2 
shows a profile of spectral width measured with the antenna at $50^{\circ}$ elevation. Two obvious differences from the vertically directed measurements are apparent, namely the variability with height, and the overall increase in width. Recall that the spectral widths measured with a vertical antenna were smaller than the model values (Fig. 2); at $50^{\circ}$ elevation they are now larger.

The left panel of Fig. 3 displays the horizontal meridional wind determined from the line-of-sight velocity estimates. The wave-like variation of the wind with altitude is related to either long-period gravity waves, or, more likely, tidal behaviour (Rietveld et al., 1992). The vertical shear in the horizontal wind is shown in the right panel of Fig. 3. It is clear that the anomalously large values of spectral width correspond to heights where the velocity shear is largest. The shears are calculated from the gate-to-gate differences in velocity. They therefore apply to altitudes midway between the nominal gate heights and the values for both velocity and velocity shear have been interpolated onto a common altitude grid with a step size of half a gate $\left(400 \mathrm{~m}\right.$ at elevation $\left.50^{\circ}\right)$

In Fig. 4 we reproduce the measured and fitted spectra for the five gates centred on $71.6 \mathrm{~km}$ altitude where the fitted spectral width is about $40 \mathrm{~Hz}$, which is a factor of four greater than that measured at the same height with vertical antenna. Above and below the centre gate there are fairly clean spectra with widths of $\sim 12 \mathrm{~Hz}$, but with a difference in Doppler shift of the order of $25 \mathrm{~Hz}$. At $71.6 \mathrm{~km}$ the two spectral components are equally strong and the fitting procedure has produced a very broad single spectrum to accom- modate the two components. In physical terms these observations indicate an abrupt change in the meridional neutral wind, from 15 to $35 \mathrm{~m} \mathrm{~s}^{-1}$ in less than $2 \mathrm{~km}$ of altitude, i.e. a shear of more than $10 \mathrm{~m} \mathrm{~s}^{-1} \mathrm{~km}^{-1}$.

The limited height resolution of the experiment does not allow more detailed investigation of these sharp velocity changes, but the vertical shear in the horizontal wind may be very large at times. For example, $\mathrm{Wu}$ et al. (1995) used radar tracking of foil clouds released from a rocket to reveal the existence of a shear of $330 \mathrm{~m}$ $\mathrm{s}^{-1} \mathrm{~km}^{-1}$ at an altitude of $87 \mathrm{~km}$. The instantaneous meridional wind at that height was $190 \mathrm{~m} \mathrm{~s}^{-1}$. This was inferred to be the manifestation of an inertial gravity wave, rather than the diurnal or semi-diurnal tide. This shear value is an order of magnitude greater than the maxima seen in the present EISCAT data.

Figure 5a shows a similar example to Fig. 3, but from a slightly later time when the antenna elevation had been lowered to $45^{\circ}$. The shear-broadening effects are even more striking here and show a high degree of correlation with the velocity shear. Note that the smallest measured values of spectral width near heights of 57 and $63-66 \mathrm{~km}$ are upper estimates due to the frequency resolution of the experiment. Although narrow spectra were not resolved, the observed Doppler shifts still give information on the average wind component within the measured volume.

The spectra from which the velocities in the left panel of Fig. 5a were determined are shown in the right panel of Fig. 5b. Close examination of Fig. 5b confirms that the peaks in the spectral width profile (Fig. 5a) indeed occur where the spectra are broadened in regions of abrupt velocity change. The broadened spectra may be

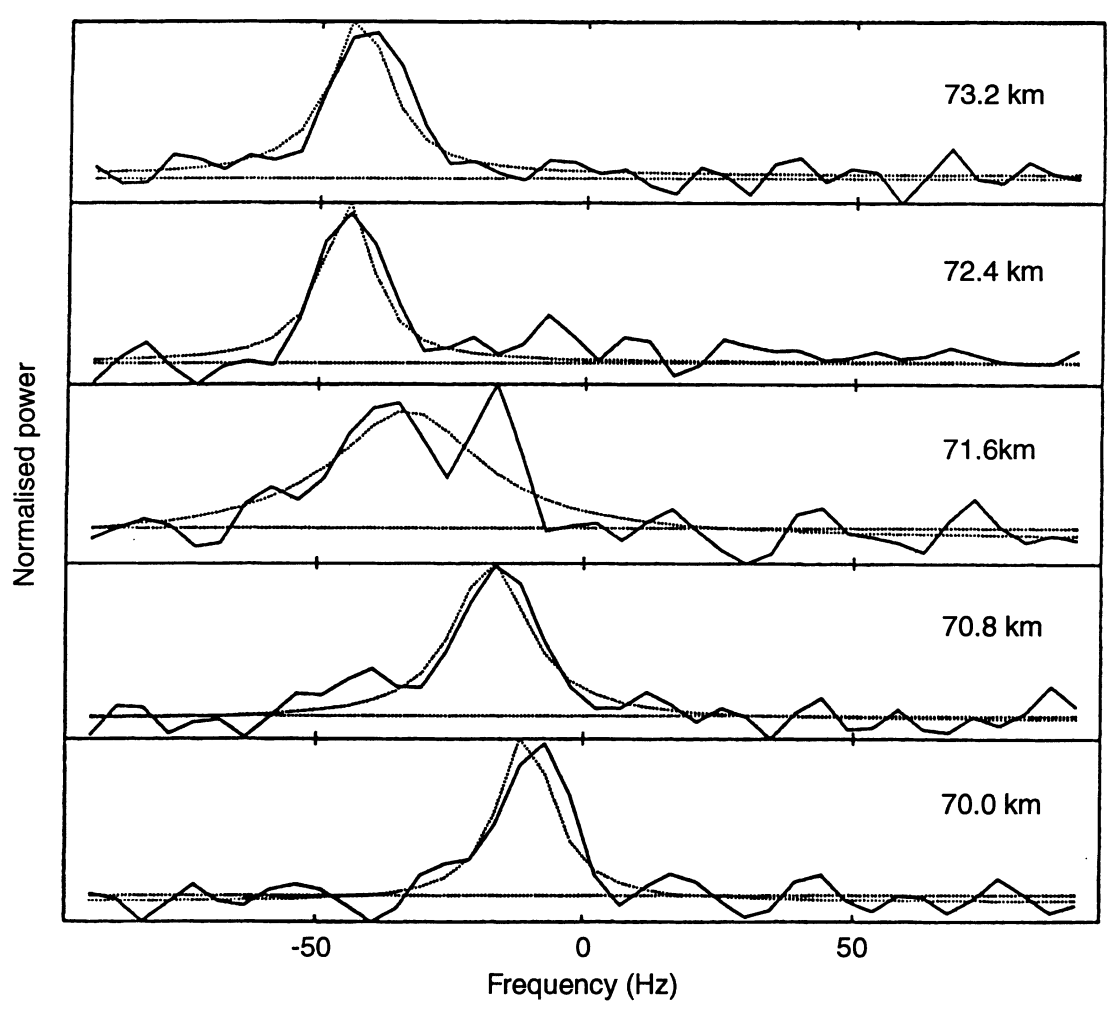

Fig. 4. Measured (full lines) and fitted (dotted lines) VHF spectra for five gates centred on $71.6 \mathrm{~km}$ altitude for the same time-interval as the results in Fig. 3 

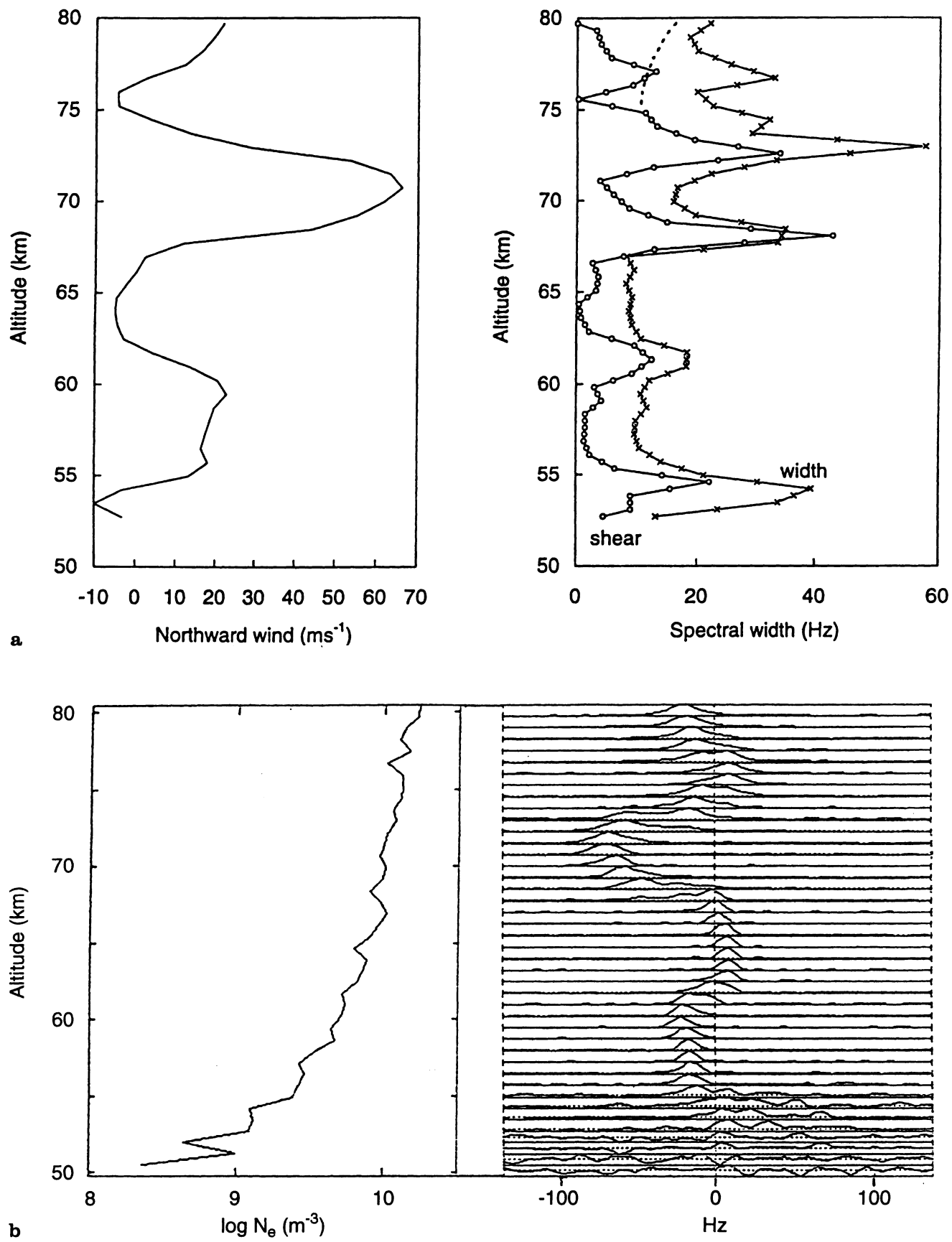

Fig. 5. a As Fig. 3, but for an antenna elevation of $45^{\circ}$ at 1330 UT. b. The right panel shows measured VHF power spectra (arbitrary linear power scale) for the same time as part a. Each spectrum is

normalised to the peak power at each height. The left panel shows the electron density profile determined from single Lorentzian fits to the spectra.

characterised as either a single, wide spectrum (at $\sim 61$ and $\sim 77 \mathrm{~km}$ in this example), or as a wider spectrum with more than one main peak (at $\sim 68$ and $\sim 73 \mathrm{~km}$ ). For the whole data set, we expect that there will be a mixture of these cases depending on whether the strongest velocity shear occurs near the upper or lower boundary of a range gate, or towards the centre, respectively. Some of the spectral broadening at heights below $\sim 70 \mathrm{~km}$ may also be attributed to the presence of negative ions, as will be demonstrated later for UHF measurements, but the effect of shear-broadening at most heights prevents detailed interpretation in terms of aeronomy.

Fitting a single analytical spectrum to an observed spectrum that contains more than one peak is clearly inadequate for aeronomical studies. However, as we have seen, the mean Doppler shift still gives valuable information about dynamics. And while we might intuitively expect that the total backscattered power estimates may be in error in such cases, this turns out not to be a serious problem. With a constant ion production rate, such as during SPE events, the number 
of scatterers (electrons) within a sampled volume should remain essentially constant, regardless of the presence, or not, of a velocity shear. Consider, qualitatively, the transition from a regime with a single, well-defined spectral component, to one with either a spread of velocities or two well-defined components. The overall spectral width will increase, but the number of scatterers at any particular Doppler shift will decrease, i.e. the overall spectral amplitude will decrease. Since the backscattered power (and thus the electron density) is proportional to both the amplitude and the width of the spectrum, this trade-off means that the electron density estimates are not seriously affected and the uncertainties are no greater than other sources of error. This is illustrated in the left panel of Fig. 5b, where the electron density profile can be seen to be relatively smooth and there are no features correlated with regions of strong shear.

\subsection{Obliquely-directed UHF radar observations}

The UHF radar was pointing in the same direction and measuring with the same pulse scheme as the VHF radar (apart from the differences in IPP discussed earlier) during the time of the observations in Fig. 5. The range-gating of the two schemes was identical, but we need to bear in mind the difference in beam dimensions between the two radars. These are circular $0.45^{\circ}$ for UHF, and $1.37^{\circ} \mathrm{N}-\mathrm{S}$ and $0.8^{\circ} \mathrm{E}-\mathrm{W}$ for VHF, to the $-3-\mathrm{dB}$ points, allowing for both transmission and reception.

Figure 6 displays the UHF results. The velocities (and velocity shears) are virtually identical with the
VHF results, but the spectral widths show much less shear-broadening. We attribute the latter characteristic to the smaller vertical extent of the UHF antenna beamwidth encompassing a smaller spread of velocities, in regions of wind shear, than the (three times) broader VHF antenna beam.

The UHF observations are close to the model values above $67 \mathrm{~km}$ but moderately constant below $67 \mathrm{~km}$, being in the range $50-75 \mathrm{~Hz}$ there. At 68 and $73 \mathrm{~km}$ there are peaks in spectral width corresponding to maxima in the velocity shear. Below $67 \mathrm{~km}$, there is some correlation between small increases in spectral width at some heights and regions of velocity shear, but the main characteristic of the UHF spectra at these heights is that they are all much broader than the model predictions. In contrast to the VHF observations, the spectral resolution applied here is not a limiting factor. Thus we can be confident that the measurement technique is reliable down to the lowest heights shown in Fig. 6.

The spectra from the lower heights in the range shown in Fig. 6 are illustrated in Fig. 7. These indicate that the measured spectra are well defined and pose no problem for the fitting algorithm. That the observed UHF widths are much larger than the model values can be accounted for by the presence of negative ions during these daytime observations, which are not included in the simple model calculations used in the foregoing. Longer time-series of spectral width changes through twilight reveal the relative variation of negative ion densities under changing solar illumination conditions (Rietveld and Collis, 1993), but more detailed investigation of the ambient negative ion chemistry requires the use of ion-chemical models (E. Turunen, 1993, 1996).
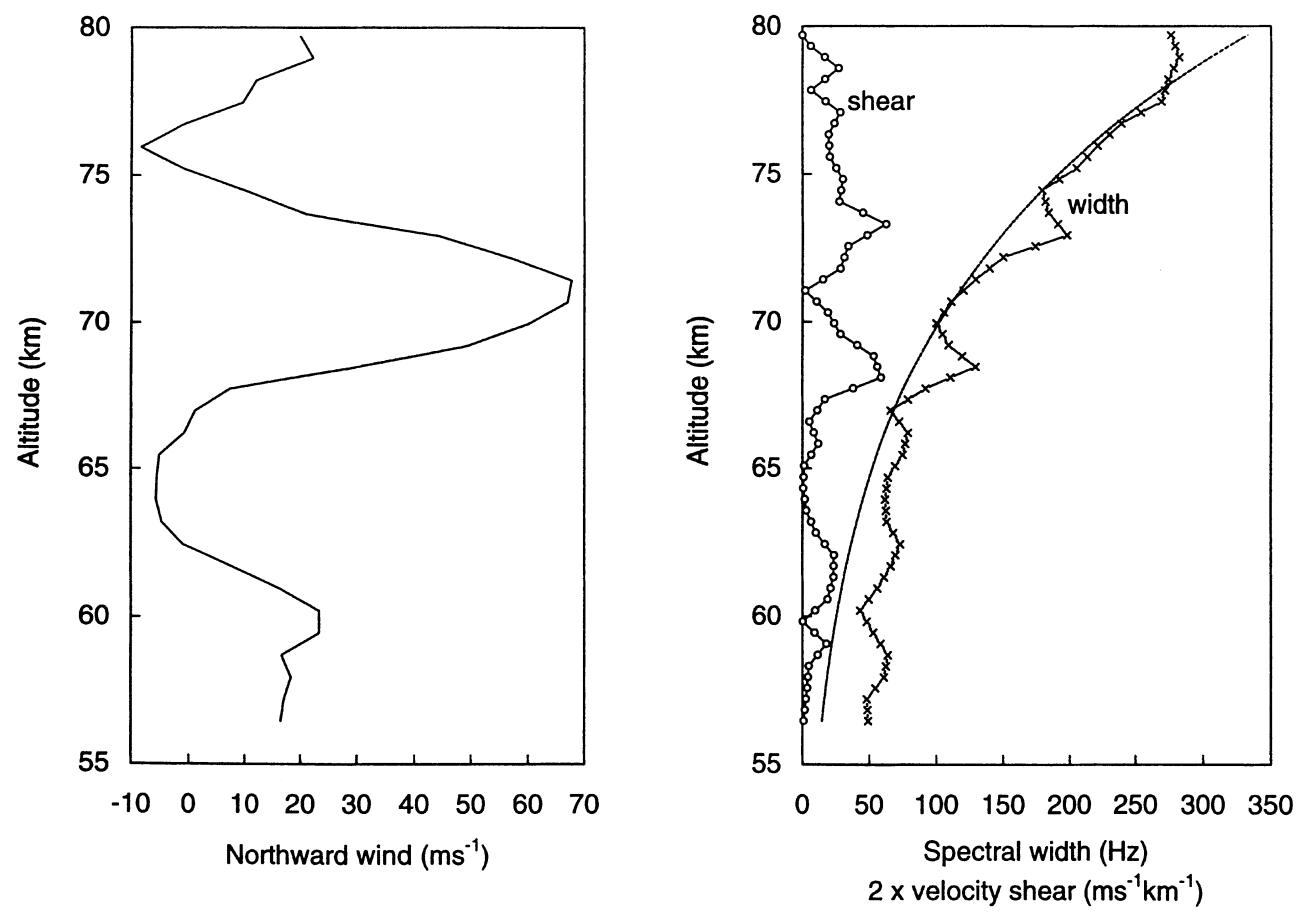

Fig. 6. As Fig. 5, but for the UHF radar. The smooth curve is the model spectral width assuming no negative ions present 


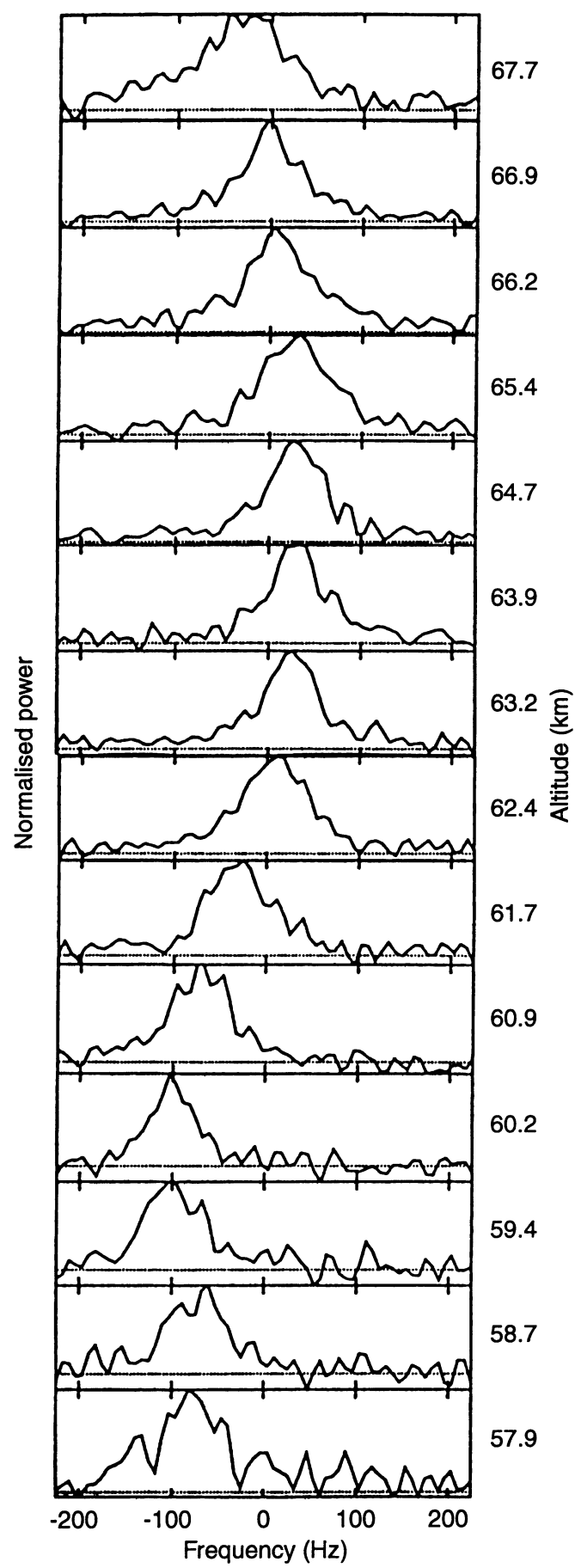

Fig. 7. Spectra measured by the UHF radar between 58 and $68 \mathrm{~km}$ altitude at an elevation angle of $45^{\circ}$ at 1330 UT on 20 March 1990

\subsection{Simultaneous UHF multipulse and VHF pulse-to- pulse observations}

At 1530 UT on 20 March, the UHF experiment was changed from the pulse-to-pulse version described earlier to one with a multipulse scheme covering upper mesosphere heights and above (CP-2-D). The VHF pulse-to-pulse operation continued throughout. CP-2 consists of a four-position scan repeated every $6 \mathrm{~min}$, with pointing directions vertical, south (S), south-east
(SE) and field-aligned. The observations by the two radars were thus no longer from the same atmospheric volumes. However, the $\mathrm{S}$ position in $\mathrm{CP}-2$ (azimuth $166.5^{\circ}$, elevation $62.9^{\circ}$ ) is useful for this study as it pointed almost directly opposite from the due-northdirected VHF beam (azimuth $359.5^{\circ}$ ). Hence both yield the meridional wind, but with a N-S horizontal separation of $120 \mathrm{~km}$ at $80 \mathrm{~km}$ altitude. The SE position of CP-2 (azimuth $133.6^{\circ}$, elevation $60.4^{\circ}$ ) may also be a useful indicator of the meridional wind, though the estimates will be less reliable if there is a significant zonal component present. The lowest height covered by the CP-2 multipulse measurements $(\sim 80 \mathrm{~km})$ matches the highest VHF gate (at elevation $45^{\circ}$ ), thereby providing continuity in altitude. The VHF elevation was raised to $58^{\circ}$ between 1922 and 2240 UT to afford some overlap in height with the CP-2 observations.

Figure 8 displays two examples of the meridional wind observed simultaneously by the UHF and VHF radars. At 1542 UT (left panel), the antenna elevations resulted in the altitudes of the highest VHF gate and the lowest UHF one being at almost the same altitude. There is no obvious continuity of the wind profile with height through the transition at $\sim 82 \mathrm{~km}$, although the two independent UHF profiles show reasonable agreement with each other. This discrepancy is perhaps not surprising given the different height resolutions of the two pulse schemes and the horizontal separation of the two sets of measurements. Indeed, the highest VHF gates and the lowest UHF ones are precisely where fitting the spectra (and thus estimating the velocity) from the two techniques is most uncertain. It should also be noted that the uncertainties in the two sets of measurements are different. The VHF results are considered very accurate for two reasons. First, the 2-min averaged profiles change only slowly with time as the phase of the long-period waves propagate vertically at about $1 \mathrm{~km} \mathrm{~h}^{-1}$ and there is little inherent noise. Second, the agreement between the VHF velocities and the UHF velocities when both radars were operating the same pulse scheme was extremely close (Sect. 4.3). The error bars are probably not more than a few $\mathrm{m} \mathrm{s}^{-1}$, comparable with the size of the plotted symbols in Fig. 8. The UHF multipulse velocities are more uncertain. The error bars vary with signal-to-noise ratio (height dependent) but are also affected in an uncertain way by short-term variations in the wind, and, for higher altitudes, in electric field. For the examples in Fig. 8, the formal error estimates from the data analysis suggest uncertainties of the order of $\pm 20 \mathrm{~m} \mathrm{~s}^{-1}$.

For the second case in Fig. 8 (right panel), there is an offset between the two UHF profiles around 100$110 \mathrm{~km}$, which could result from the presence of an eastward wind of magnitude several tens $\mathrm{m} \mathrm{s}^{-1}$ in this region. We therefore consider the results from the $\mathrm{S}$ position to be the more reliable here. Importantly, however, the higher elevation of the VHF antenna provided some overlap in height between the UHF and VHF measurements, and the profiles can be seen to match smoothly. In this case the two sets of observations could be combined and used with some confidence 


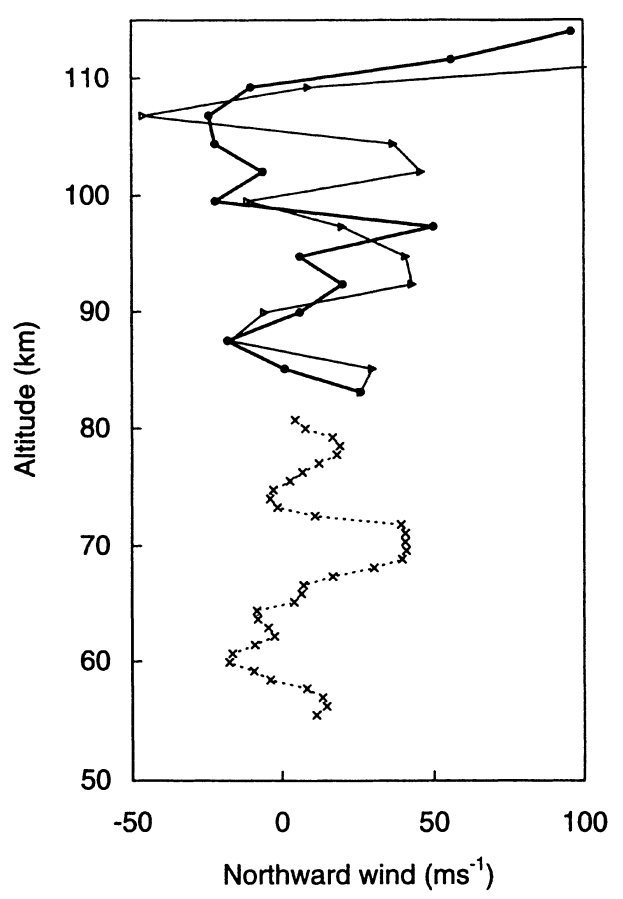

Fig. 8. Two examples of horizontal meridional wind measured simultaneously by the UHF and VHF radars on 20 March 1990. The VHF profiles are from a 2-min integration while the UHF results are averages over 70-s dwells in four successive 6-min scans (individual UHF profiles tend to be noisy due to the lower snr). The meridional wind derived from the $\mathrm{S}$ position $(\mathbf{O})$ will be more

to investigate mesosphere and lower thermosphere dynamics. Future studies using such experiments should allow for some overlap in altitude coverage to avoid potential uncertainties in the transition region caused by results such as those shown in the left panel of Fig. 8.

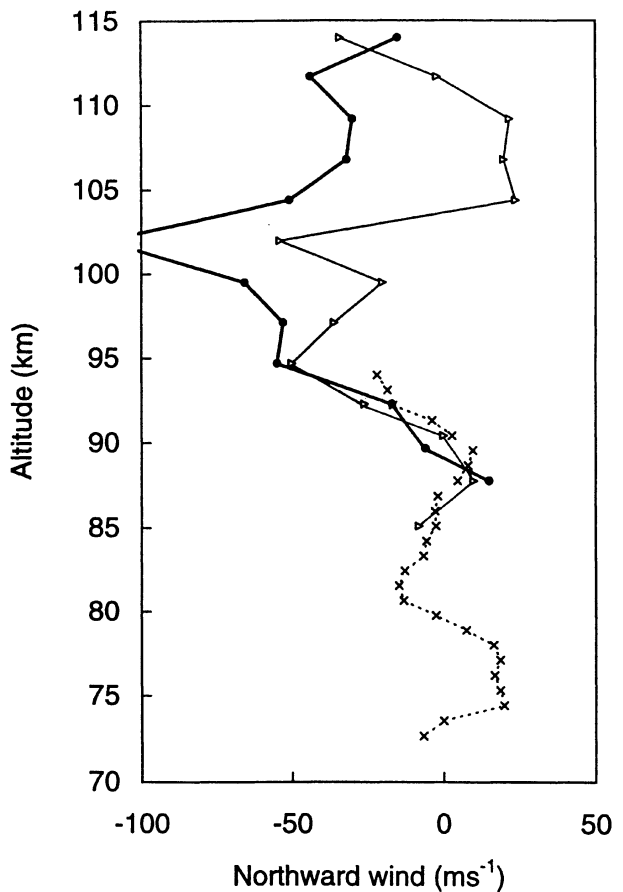

reliable than that from the SE position $(\triangle)$ if there is a significant zonal wind present. The uncertainties for the VHF results are no more than a few $\mathrm{m} \mathrm{s}^{-1}$ while for the UHF they are probably at least $\pm 20 \mathrm{~m}$ $\mathrm{s}^{-1}$ (see text). Left: VHF at elevation $45^{\circ}(-\mathrm{x}-), 1542 \mathrm{UT}$. Right: VHF at elevation $58^{\circ}(-\mathrm{x}-), 2000 \mathrm{UT}$

\subsection{UHF multipulse and pulse-to-pulse observations}

Multipulse and pulse-to-pulse modulations cannot be transmitted simultaneously within the same pulse scheme with the EISCAT radars. The value of consid-
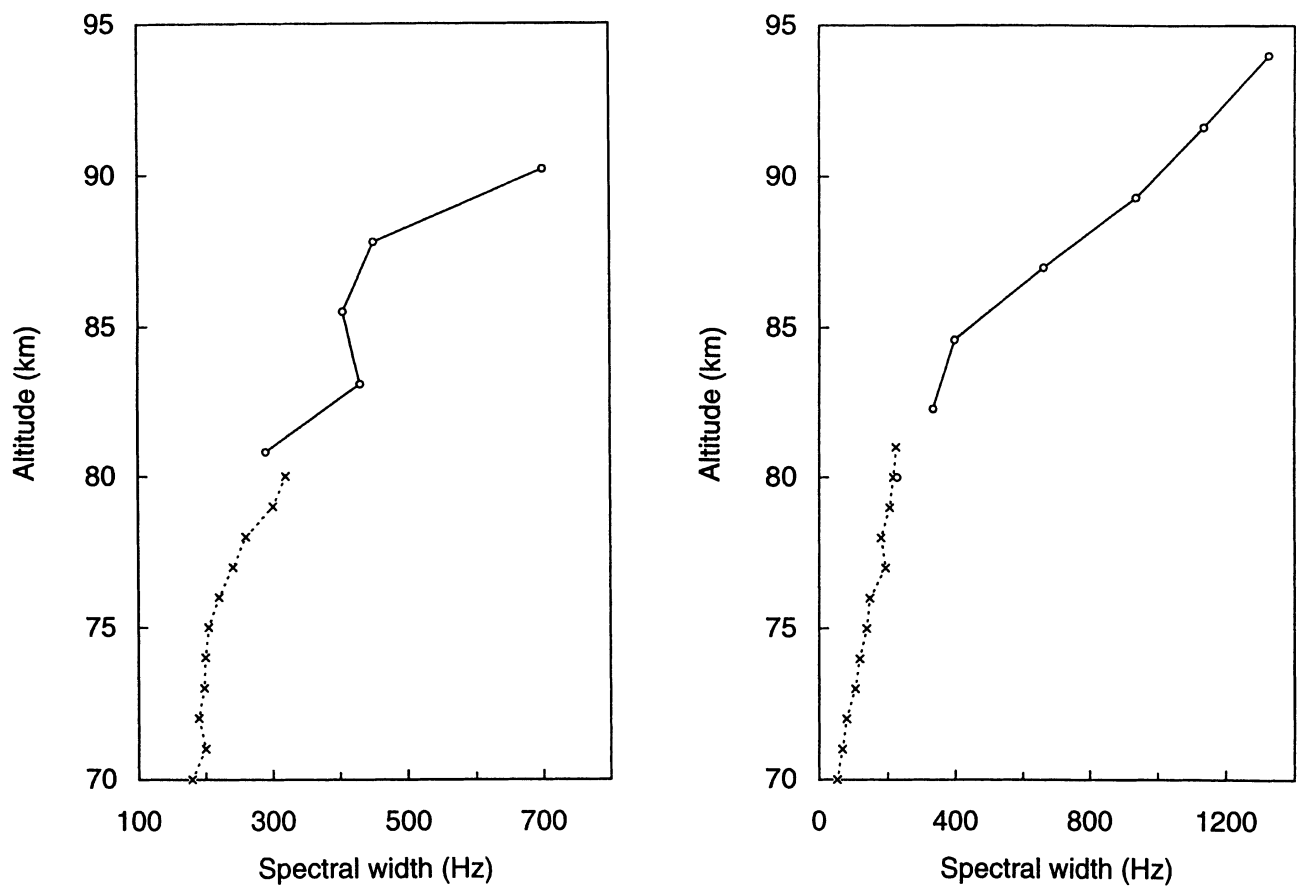

Fig. 9. Synthesis of spectral width measurements at UHF during PCA events from multipulse (o) and pulse-to-pulse (-x-) observations on the same day. Left: 23 October 1989, 1530 UT (x) and 1803 UT (o). Right: 1 December 1989, 1910 UT (x) and 0917 UT (o) 

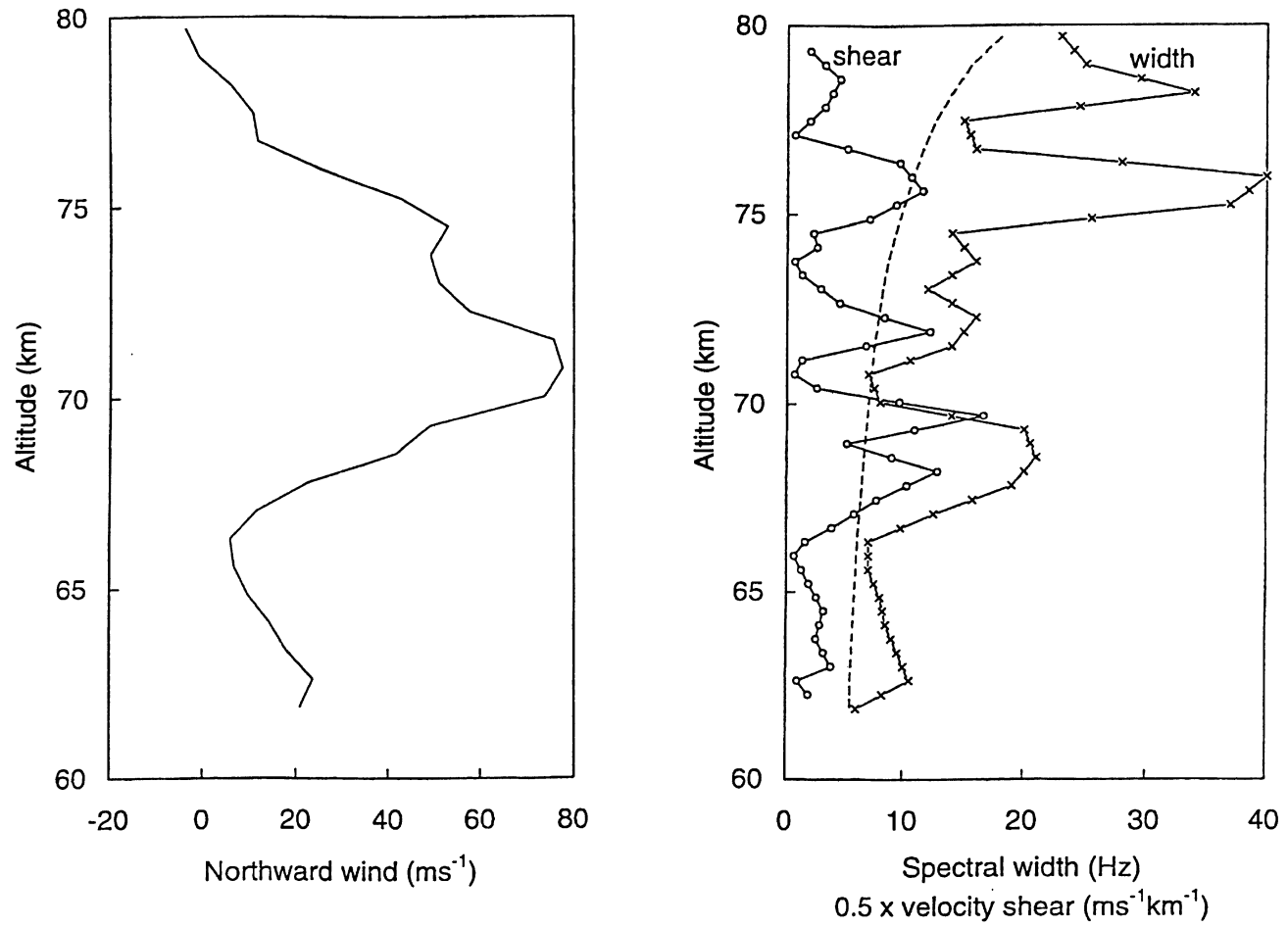

Fig. 10. As Fig. 5, but with an IPP of $6.666 \mathrm{~ms}$. The measurements are from 0905 UT on 21 March 1990

ering both pulse schemes together is that a wide altitude range, from the mesosphere to the thermosphere, can be studied, which cannot be satisfied by any individual pulse scheme. One possibility is to design an experiment that switches automatically between the two schemes every few minutes (Rietveld and Collis, 1993). The approach illustrated here, however, is to consider observations of the same PCA event by the two schemes at different times on the same day.

We examine just spectral widths here, and do not attempt any detailed interpretation because of the nonsimultaneity of the measurements. Figure 9 shows two such pairs of results from the SPE events on 23 October and 1 December 1989. The fact that both pairs of profiles overlap smoothly gives confidence in the measurement techniques and the analysis methods for both schemes at these heights. This also adds further weight to the real nature of the discrepancy between observed and measured spectral widths in the upper mesosphere mentioned in Sect. 4.1.

\subsection{VHF pulse-to-pulse spectra with increased frequency resolution}

The VHF observations above (in Sects. 4.1 and 4.2) had $7-\mathrm{Hz}$ frequency resolution, resulting from 43 measured $\mathrm{ACF}$ lags at a spacing of $3.24 \mathrm{~ms}$. Better frequency resolution can be achieved by increasing the longest measured lag in the ACF. In principle, this could be accomplished by increasing the number of lags while retaining the same lag spacing. However, with the presently available experiments at EISCAT, the practical solution is to increase the IPP in GEN-11. This has the drawback of reducing the radar duty cycle, and thus the overall signal-to-noise ratio, but for PCA events, with their large electron densities, this may not be too disadvantageous.

A pulse-to-pulse scheme with an increased IPP of $6.666 \mathrm{~ms}$ was tested on 21 March 1990, though the Dregion electron densities were by then significantly less than on the first day of measurements. The spectra were thus noisier than those from 20 March, shown in Fig. 4 for example (or the UHF spectra in Fig. 7). Even under these conditions, however, the mean Doppler shift was accurately found by the fitting algorithm and the resultant velocity patterns matched seamlessly with those before and after in which the lower IPP value was used. The spectral widths were also fitted relatively well, though as the antenna elevation was $45^{\circ}$ the spectra were badly affected by shear broadening as described earlier. Figure 10 shows the velocity, velocity shear and spectral widths for a 5-min post-integration from this period. The minima in the observed spectral widths (i.e. when not affected by shear broadening) do come close to the model values at some heights. It would have been even more interesting to compare the observations with the model for altitudes lower than $62 \mathrm{~km}$, as shown in Fig. 5 for 20 March, but the electron densities at these heights on 21 March were too small to allow this.

\section{Summary and conclusions}

Many of the EISCAT operations aimed at mesospheric measurements during SPE events in solar cycle 22 were truly exploratory in nature. The scope of this paper has been principally to examine the technical aspects of such 
applications, which follow in Sect. 5.2 below. We first summarise the scientific results from the present investigation.

\subsection{Scientific results}

One scientific result to emerge from this work was the observation by the VHF radar of spectral widths that are significantly smaller than model predictions, at least in the 76-96-km altitude range. This provides independent confirmation of earlier results from the EISCAT UHF radar (Hansen et al., 1991; Rietveld and Collis, 1993) and suggests that radar frequency is not a factor in explaining the discrepancy. We were not able accurately to determine the spectral widths below $\sim 76 \mathrm{~km}$ due to the frequency resolution of the experiment.

Our approach of combining EISCAT UHF and VHF incoherent-scatter observations that overlap near the mesopause is also new. The spectral width results demonstrate that the measurement techniques and analysis methods are reliable, with the exception of spectral width determination when the VHF radar points away from vertical (see Sect. 5.2). The velocity measurements indicate the possibility of observing atmospheric dynamics continuously through the mesosphere and lower thermosphere with good height and time resolution. Initial comparisons of the UHF and VHF results show that the wave-like nature of the wind variations with height (presumably tidal in origin) can be successfully compared and combined provided that there is some overlap in the altitude coverage of the two schemes. Combined observations that leave a gap in altitude coverage can result in discontinuities that are difficult to reconcile. The horizontal separation of $\sim 100 \mathrm{~km}$ between the sampled UHF and VHF volumes in the experiments reported here may also exacerbate the comparison and future studies should use collocated observations.

\subsection{Technical Aspects}

The principal technical result of this work was the demonstration of severe spectral broadening in the VHF radar data when the antenna was tilted substantially away from vertical. This effect prevents the use of most of the spectral measurements for aeronomical applications, but the mean Doppler shifts provide valuable information on neutral winds. The winds determined from both the UHF and VHF systems were virtually identical when viewing the same ionospheric volumes simultaneously. This is a new result and a confirmation of the value of the technique for the study of atmospheric dynamics.

UHF spectral observations made with oblique antenna pointing were affected by wind shear to a smaller extent than the VHF spectra. This is due to the narrower UHF beam spanning a smaller height range than the VHF, thereby encompassing a smaller range of velocities in regions of vertical shear in the horizontal wind.
Thus although the lower operating frequency of the VHF system is in principle more effective than the UHF for mesospheric observations, the practical implementation is less useful. The VHF may even be sensitive to turbulence scatter from the middle mesosphere during PCA conditions (Collis et al., 1992) and is certainly subject to strong non-incoherent backscatter from the upper mesosphere in summer (e.g. Cho and Röttger, 1997, and references therein).

The frequency resolution of the GEN-11 experiment scheme used for the VHF observations was not sufficient to resolve spectra below about $76 \mathrm{~km}$ altitude. However, the Doppler shift could still be estimated and neutral winds determined. A version of the VHF experiment with improved frequency resolution produced encouraging results, but the test conditions were not optimum for this scheme at the lowest altitudes. This higher IPP modulation is probably the best available candidate for observations with a vertical, or near-vertical, VHF antenna when the next major SPE erupts.

Acknowledgements. The EISCAT Scientific Association is supported by Suomen Akatemia of Finland, Centre National de la Recherche Scientifique of France, Max-Planck-Gesellschaft of Germany, the National Institute of Polar Research of Japan, Norges Forskningsråd of Norway, Naturvetenskapliga Forskningsrådet of Sweden, and the Particle Physics and Astronomy Research Council of the United Kingdom.

Topical Editor D. Alcayde thanks P. J. S. Williams and A. D. Aylward for their help in evaluating this paper.

\section{References}

Bauer, P., Theory of waves incoherently scattered, Phil. Trans. Roy. Soc., A280, 167-191, 1975.

Beynon, W. J. G. and P. J. S. Williams, Incoherent scatter of radio waves from the ionosphere, Rep. Prog. Phys., 41, 909-956, 1978.

Cho, J. Y. N., and J. Röttger, An updated review of polar mesosphere summer echoes: observation, theory, and their relationship to noctilucent clouds and subvisible aerosols, $J$. Geophys. Res., 102, 2001-2020, 1997.

Collis, P. N. and M. T. Rietveld, Mesospheric observations with the EISCAT UHF radar during polar cap absorption events: 1 . Electron densities and negative ions, Ann. Geophysicae, 8, 809$824,1990$.

Collis, P. N., and J. Röttger, Mesospheric measurements with the EISCAT UHF and VHF radars: a review of principles and experimental results, J. Atmos. Terr. Phys., 52, 569-584, 1990.

Collis, P. N., and J. Röttger, Correction to "Mesospheric measurements with the EISCAT UHF and VHF radars: a review of principles and experimental results", J. Atmos. Terr. Phys., 53, 463, 1991.

Collis, P. N., T. Turunen and E. Turunen, Evidence of heavy positive ions at the summer arctic mesopause from the EISCAT UHF incoherent- scatter radar, Geophys. Res. Lett., 15, 148$151,1988$.

Collis, P. N., M. T. Rietveld, J. Röttger, and W. K. Hocking, Turbulence scattering layers in the middle-mesosphere observed by the EISCAT $224 \mathrm{MHz}$ radar, Radio Sci., 27, 97-107, 1992.

Evans, J. V., Theory and practice of ionosphere study by Thomson scatter radar, Proc. IEEE, 57, 496-530, 1969.

Hall, C., U.-P. Hoppe, P.J.S. Williams, and G. O. L. Jones, Mesospheric measurements using the EISCAT VHF system: first results and their interpretation, Geophys. Res. Lett., 14, 1187-1190, 1987. 
Hansen, G., U-P. Hoppe, E. Turunen, and P. Pollari, Comparison of observed and calculated incoherent-scatter spectra from the D-region, Radio Sci., 26, 1153-1164, 1991.

Kofman, W., and C. Lathuillere, EISCAT multipulse technique and its contribution to auroral ionosphere and thermosphere description, J. Geophys. Res., 90, 3520-3524, 1985.

Kofman, W., F. Bertin, J. Röttger, A. Cremieux, and P. J. S. Williams, The EISCAT mesospheric measurements during the CAMP campaign, J. Atmos. Terr. Phys., 46, 565-575, 1984.

Mathews, J. D., Incoherent-scatter radar probing of the $60-100-\mathrm{km}$ atmosphere and ionosphere, Proc. IEEE Trans. Geosc. Rem. Sensing, 24, 765-776, 1986.

Rietveld, M. T., and P. N. Collis, Mesospheric observations with the EISCAT UHF radar during polar cap absorption events: 2 . spectral measurements, Ann. Geophysicae S, 11, 797-808, 1993.

Rietveld M. T., P. N. Collis and J. Röttger, Observations of atmospheric dynamics in the low- and middle-mesosphere with the EISCAT incoherent-scatter radar during solar proton events, Adv. Space Res., 12, (10)81-(10)84, 1992.

Solar Geophysical Data, Preliminary report and forecast of, SESC PRF 760, NOAA, 325 Broadway, Boulder, Colorado 803033328, USA, 1990.
Turunen, E., EISCAT incoherent-scatter radar observations and model studies of day to twilight variations in the D-region during the PCA event of August, 1989, J. Atmos. Terr. Phys. 55, 767-781, 1993.

Turunen, E., Incoherent-scatter radar contributions to high-latitude D-region aeronomy, J. Atmos. Terr. Phys., 58, 707-725, 1996.

Turunen, E., P. N. Collis, and T. Turunen, Incoherent-scatter spectral measurements of the summertime high-latitude Dregion with the EISCAT UHF radar, J. Atmos. Terr. Phys., 50, 289-299, 1988.

Turunen, T., GEN-SYSTEM - a new experimental philosophy for EISCAT radars, J. Atmos. Terr. Phys., 48, 777-785, 1986.

Turunen, T., and J. Silén, Modulation patterns for the EISCAT incoherent scatter radar, J. Atmos. Terr. Phys., 46, 593-599, 1984.

Wu, Y.-F., H.-U. Widdel, and D. Offerman, First observation of a mesospheric wind shear as high as $330 \mathrm{~m} \mathrm{~s}^{-1} \mathrm{~km}^{-1}$, Ann. Geophysicae, 13, 954-958, 1995. 